\title{
The Influence of Wrist Posture, Grip Type, and Grip Force on Median Nerve Shape and Cross-sectional Area
}

\begin{abstract}
(4)
ABSTRACT (249 WORDS)

Introduction: During grasping, the median nerve undergoes mechanical stress in the carpal tunnel which may contribute to carpal tunnel syndrome. This study investigated the effects of wrist posture, grip type, and grip force on the shape and cross-sectional area of the median nerve. Materials and Methods: Ultrasound examination was used to obtain cross-sectional images of the dominant wrist of 16 healthy subjects ( 8 male) at the proximal carpal tunnel during grasping. The cross-sectional area, circularity, and axis lengths of the median nerve were assessed in 27 different conditions ( 3 postures x 3 grip types x 3 force levels). Results: There were no significant changes in median nerve cross-sectional area $(P>0.05)$. There were significant interactions across posture, grip type, and grip force affecting nerve circularity and axis lengths. When the wrist was flexed, increasing grip force caused the median nerve to shorten in the mediolateral direction and lengthen in the anteroposterior direction $(P<0.04)$, becoming more circular. These effects were significant during four finger pinch grip and chuck grip $(P<0.05)$ but not key grip $(P>0.07)$. With the wrist extended, the nerve became more flattened (less circular) as grip force increased during four finger pinch grip and chuck grip $(\mathrm{p}<0.04)$ but not key grip $(\mathrm{p}>0.3)$. Circularity was lower during the four finger pinch compared to chuck or key grip $(\mathrm{p}<0.03)$. Conclusions: The findings suggest that grip type and wrist posture significantly alter the shape of the median nerve.
\end{abstract}

Key Words: Median Nerve, Carpal Tunnel Syndrome, Ultrasound, Wrist, Grip

This is the author manuscript accepted for publication and has undergone full peer review but has not been through the copyediting, typesetting, pagination and proofreading process, which may lead to differences between this version and the Version record. Please cite this article as doi:10.1002/ ca.22871. 


\section{INTRODUCTION}

The carpal tunnel is a narrow passage at the wrist formed by the carpal bones and the transverse carpal ligament. Nine tendons and the median nerve pass through the carpal tunnel (Presazzi et al., 2011) During finger activity, the median nerve and digital flexor tendons move longitudinally and transversely in the carpal tunnel (Filius et al., 2015; van Doesburg et al., 2012). This can cause pressure (Keir et al., 2007), deformation (van Doesburg et al., 2010), and friction (Kociolek et al., 2015) on the median nerve. The resulting contact stresses between the median nerve and adjacent structures are a probable source of pathogenesis (Ko and Brown, 2007). Furthermore, abnormal nerve movement in the carpal tunnel may be a sign of the development or progression of carpal tunnel syndrome (Filius et al., 2015).

Carpal tunnel syndrome (CTS) is a common peripheral neuropathy of the upper extremity which affects nearly $8 \%$ of industrial workers (Dale et al., 2013). While the exact etiology of CTS is unknown, animal models (Clark et al., 2004) and epidemiological studies (Armstrong et al., 2008; Bonfiglioli et al., 2007; Stapleton, 2006) indicate that forceful, repetitive grasping is a major risk factor for CTS. To better understand how different tasks affect CTS risk, researchers have examined the volume and pressure in the carpal tunnel using magnetic resonance imaging or percutaneous catheters. Results demonstrate that deviating from neutral wrist posture decreases the volume (Mogk and Keir, 2009) and increases the pressure (Keir et al., 2007) in the carpal tunnel. Similarly, carpal tunnel pressure increases with increasing fingertip (Keir et al., 1998) or grip forces (Goss and Agee, 2010; McGorry et al., 2014). While these results support epidemiological study findings, the cause of nerve injury remains unclear as neither pressure nor MRI measurements can capture tendon and nerve deformations during active tasks associated with CTS. 
Advances in ultrasonography have made it possible to noninvasively measure tendon and nerve deformation in vivo. The spatial resolution of ultrasonography allows for the hyperechoic border of the median nerve to be distinguished from the tendons within the carpal tunnel. Ultrasonography also has the temporal resolution to examine the deformation of the median nerve and surrounding tendons during active grasping in a manner that is not feasible with pressure or MRI measurements of passive tasks. Ultrasound studies have shown that the median nerve is displaced by the surrounding tendons during isolated finger (Nakamichi and Tachibana, 1992; van Doesburg et al., 2010; Yoshii et al., 2009) and wrist (Lopes et al., 2011) movements. Furthermore, the median nerve cross-sectional area decreases and the nerve deforms during wrist flexion or extension (Loh and Muraki, 2015), finger flexion (Filius et al., 2015; van Doesburg et al., 2010; Yoshii et al., 2009), and prehension (Sucher, 2009). The observed deformation and reduced area of the median nerve suggests that it is subject to repetitive compression and other mechanical stress from tendons and surrounding structures during hand movements.

Typical work tasks are much more complex than the isolated (passive) wrist or finger movements examined in prior ultrasound studies. Epidemiology (Palmer et al., 2007) and cadaver (Kociolek et al., 2015) studies suggests that the combination of wrist flexion or extension with forceful gripping poses a particularly high risk of carpal tunnel syndrome. Imaging the median nerve during complex activities that combine wrist flexion/extension with active grasping may help to better understand mechanisms of nerve injury. This could help to identify people at risk of injury and to design tasks that reduce the risk of carpal tunnel syndrome. Therefore, the purpose of this study was to examine the effects of wrist posture, grip type, and grip force on median nerve cross-sectional area and circularity in healthy subjects. Three pinch grip positions (Fig. 1 A-C) were selected because pinching is a common 
occupational activity, results in high tendon forces (Armstrong and Chaffin, 1979) and has been implicated in the development of CTS (Silverstein et al., 1987). Moreover, the tendons directly adjacent to the median nerve (flexor pollicis tendon and the flexor tendons of the second and third digits) are utilized differently in each grip type (An et al., 1985; Li et al., 1998). Three wrist postures (neutral, 30 degrees flexion, and 30 degrees extension) were selected based on previous work demonstrating compression of the median nerve with deviations from neutral wrist position (Loh and Muraki, 2014). We hypothesized that deformation of the median nerve would increase in non-neutral wrist postures and that these changes would be dependent on grip type and grip force.

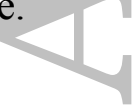

\section{METHODS}

Subjects

Seventeen (17) healthy, right-handed subjects ( $22 \pm 2$ years; 8 female) participated in this institutionally approved study after providing their written informed consent. Potential subjects were screened to ensure they did not have any symptoms of CTS. Screening included the Boston Carpal Tunnel questionnaire (Levine et al., 1993), Phalen's test (Signs-CTS, 2000), and Tinel's test (Tinel, 1945). Exclusion criteria included an average score $>1.5$ or any answer $>2$ on the Boston Carpal Tunnel questionnaire, or positive signs (numbness, tingling, or pain in the hand or wrist) for either Phalen's or Tinel's tests. Data for one subject was eliminated from the analysis due to the presence of a bifurcated nerve which prevented consistent identification of the entire nerve in all conditions. The remaining data for 8 males and 8 females was analyzed.

\section{Experimental Protocol}


Each subject completed a single day of testing. During all trials, subjects sat in a chair with their forearm supinated and strapped to an armrest. The height of the armrest was adjusted until the elbow was flexed to approximately 60 degrees and the hand extended beyond the edge of the arm rest. A researcher demonstrated each grip type for the subject prior to testing. Maximum grip strength was then obtained during maximum voluntary contractions in each of nine posture/grip conditions (three wrist postures $\times$ three grips). Prior to each strength measurement, the wrist was placed in the correct position using a handheld goniometer. An electronic handheld dynamometer (Vernier, Beaverton, OR) was used to measure strength and monitor force during all trials. The same aperture was used for all participants.

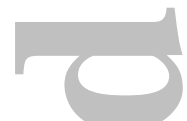

[INSERT FIGURE 1 ABOUT HERE.]

Subjects performed a series of gripping trials while B-mode ultrasound images of the median nerve were captured using an SL-15 ultrasound transducer (Aixplorer ultrasound system, Supersonic Imagine, Aix-en-provence, France). A total of 90 images were obtained from each subject. Three sets of gripping at three force levels were completed in each of the nine conditions ( 3 wrist postures x 3 grip types). Additionally, three images were obtained in each wrist posture with the fingers in a neutral position (no grip). The subject's wrist was placed in the appropriate position using a handheld goniometer. The subject then actively maintained the wrist position during all trials. The ultrasound transducer was positioned along the distal wrist crease at the level of the pisiform and scaphoid tubercle and held at an angle of approximately 90 degrees to the forearm to obtain cross-sectional images of the proximal carpal tunnel. In each condition, subjects were instructed to hold the dynamometer with minimal grip force $(\sim 0 \mathrm{~N})$. Once a clear 
image of the median nerve was obtained, the ultrasound transducer was held in approximately the same position while subjects performed gripping trials at $0 \%(\sim 0 \mathrm{~N}), 25 \%$, and $50 \%$ of maximum force. Subjects maintained grip force at each level while an image was recorded $(\sim 2$ seconds). They viewed a digital feedback display of their force output on a computer monitor during all trials. Post hoc examination of the force data indicated that each subject produced volitional forces with an error of less than $15 \%$ of the target force. An image was recorded at the first force level before subjects proceeded to grip at each subsequent force level. Following each set of force trials, the ultrasound transducer was removed and repositioned using the same anatomical landmarks. When a clear image of the median nerve was obtained, the next set of force trials was collected. The same ultrasound operator obtained all images. The order of the conditions was randomized, and the order of grip force levels alternated between increasing or decreasing force for each set of trials.

\section{Data And Statistical Analyses}

The images were transferred from the ultrasound system and examined using ImageJ (Schneider et al., 2012). The cross-sectional area (CSA) of the median nerve was calculated by tracing the hyperechoic boundary of the median nerve (Fig. 2) (Alemán et al., 2008). Median nerve deformation was assessed using three metrics. The mediolateral (ML) and anteroposterior (AP) axis diameters of the nerve were measured as the longest diameter of the nerve in the mediolateral and anteroposterior direction, respectively (Duncan et al., 1999). The circularity (Cox, 1927) of the median nerve was calculated according to:

$$
\text { circularity }=4 \pi\left({\text { Area } \left./ \text { Perimeter }^{2}\right)}_{\text {Eq. } 1 .}\right.
$$


A circularity of 1 indicates the median nerve has a perfectly circular shape, while a median nerve that is primarily elongated along the ML or AP axis will have a circularity approaching 0.

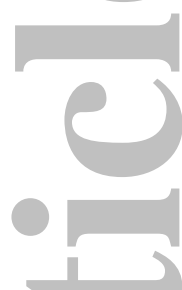

\section{[INSERT FIGURE 2 ABOUT HERE.]}

Two researchers performed the measurements on each image. The average and standard deviation of three images were obtained for each condition, and an intraclass correlation, ICC (2, 1), model was used to assess inter-rater reliability. To assess intra-rater reliability, a random selection of 50 conditions including at least three conditions from each subject was measured twice by the same researcher and analyzed using an ICC $(2,1)$ model. An ICC between 0.4 and 0.75 was considered good, and an ICC $>0.75$ was considered excellent (Fleiss, 1999).

A two-factor repeated measures ANOVA was used to assess differences in maximum voluntary grip strength between wrist postures (neutral, flexed, extended) and grip types (four finger pinch, chuck, key). The averaged CSA, circularity, and axis diameters across two raters were used as outcome measures for all statistical analyses. Differences in median nerve CSA, circularity, and diameter were assessed using three-factor repeated measures ANOVAs with wrist posture, grip type, and grip force $(0 \%, 25 \%, 50 \%)$ as factors. If a significant wrist posture $\times$ grip type $\times$ grip force interaction was found, follow-up assessments were performed using twofactor (wrist posture $\times$ grip force) repeated measures ANOVAs to assess the effects of wrist posture and grip force separately for each type of grip. Mauchly's test of sphericity was used to test for equal variance, and a Huynh-Feldt correction was applied if unequal variance was found. Significant interactions were explored using estimated marginal means. A Sidak correction for 
multiple comparisons was used for post hoc tests. All statistical tests were performed in SPSS v.22 (IBM Corporation, Chicago, IL, USA) with a level of significance of $P<0.05$.

\section{RESULTS}

\section{Grip Forces}

The maximum voluntary grip forces produced across the various postures and grip types were on average highest in the wrist neutral position and in the key grip (Fig. 3). However, there was a significant wrist posture $\times$ grip type interaction for maximum grip force $(P=0.005)$. In the four finger pinch grip and key grip, peak grip force was lower when the wrist was flexed compared to extended or neutral postures $(P<0.04)$. In the chuck grip, MVC was lower when the wrist was flexed compared to neutral $(\mathrm{p}=0.001)$ but not extended posture $(P=0.069)$. There was no difference in force between wrist neutral and wrist extended for the four finger pinch $(P$ $=0.345)$, chuck $(P=0.071)$, or key grips $(P=1)$.

\section{[INSERT FIGURE 3 ABOUT HERE.]}

\section{Reliability}

Intra-rater reliability was excellent, and inter-rater reliability was good for median nerve circularity (Table 1). Intra-rater and inter-rater reliability for cross-sectional area (CSA), mediolateral (ML), and anteroposterior (AP) diameters were excellent (Table 1). The average standard deviation of three images was $0.343 \mathrm{~mm}^{2}$ (range: $0.374-1.254$; Fig. 4) for CSA, 0.488 mm (range: $0.026-2.376$; Fig. 5) for ML diameter, 0.25 (range: $0.024-1.613$; Fig. 5) for AP diameter, and 0.061 (range: $<0.001-0.278$; Fig. 6) for circularity (Table 2). 


\section{[INSERT TABLE 1 ABOUT HERE]}

\section{Median Nerve CSA}

There were no significant main effects for wrist posture, grip type, or grip force and no significant interactions between any of these factors on median nerve CSA $(P>0.4$; Fig. 4).

\section{[INSERT FIGURE 4 ABOUT HERE.]}

\section{Median Nerve Diameter}

The three-way ANOVA for the mediolateral (ML) axis showed main effects of wrist posture $(P=0.001)$ and grip force $(P<0.001)$ but not grip type $(P=0.262)$. There was also a significant wrist posture $\times$ grip type $\times$ grip force interaction effect $(P=0.01)$. Follow-up twoway ANOVAs showed wrist posture $\times$ grip force interactions during four-finger pinch $(P<$ $0.001)$ and chuck grip $(P=0.007)$ but not key grip $(P=0.768)$. Post hoc tests showed that during four-finger pinch and chuck grip the ML axis was shorter at 25 and $50 \%$ force than $\sim 0 \%$ force when the wrist was flexed $(P<0.04)$ but there was no difference in the wrist extended or neutral postures $(P>0.06$; Fig. 5A, 5B). For key grip there was a main effect of force $(P<0.001)$. The ML axis was shorter at 25 and $50 \%$ force than $\sim 0 \%$ force during key grip in all wrist postures ( $P$ $<0.003$; Fig. 5C; Table 2).

The three-way ANOVA for the anteroposterior (AP) axis showed main effects of wrist

posture $(P<0.001)$ and grip force $(P<0.001)$ but not grip type $(P=0.504)$. The three-way interaction was not significant $(P=0.079)$, but there was a significant wrist posture $\times$ grip type $(P=0.01)$ interaction effect. Post hoc tests showed that the AP axis was longer when the wrist was flexed compared to neutral or extended posture for four finger pinch $(P<0.001$; Fig. 5D) and chuck grip $(P<0.01$; Fig. 5E). For key grip, the AP axis was longer when the wrist was flexed compared to wrist neutral $(P=0.014)$. There was also a trend for the AP axis to be longer 
when the wrist was flexed compared to wrist extended $(P=0.05$; Fig. 5F). There was a wrist posture $\times$ grip force $(P<0.001)$ interaction effect. When the wrist was flexed, the AP axis was longer at $25 \%$ than 0 force $(P<0.001)$ and longer at $50 \%$ than $25 \%$ force $(P=0.037)$. Grip force did not affect AP axis length in wrist neutral $(P>0.13)$ or extended posture $(P>0.85$; Fig. 5; Table 2).

[INSERT FIGURE 5 ABOUT HERE.]

[INSERT TABLE 2 ABOUT HERE]

\section{Median Nerve Circularity}

The ANOVA for median nerve circularity showed significant main effects of wrist posture $(P<0.001)$, and force $(P=0.0361)$, and a significant three-way interaction $(P=0.015)$. Follow-up two-way ANOVAs revealed significant wrist posture $\times$ grip force interactions during four-finger pinch $(P<0.001)$ and chuck grips $(P=0.01)$ but not key grip $(P=0.882)$. During four-finger pinch, the nerve was more circular at $25 \%$ and $50 \%$ grip force compared to $\sim 0 \%$ when the wrist was flexed $(P<0.02)$, but the nerve was less circular at 25 and $50 \%$ force when the wrist was extended $(P<0.05$; Fig. 6A). During chuck grip, the nerve was more circular at $50 \%(P=0.021)$ but not $25 \%$ force $(P=0.066)$ when compared to $\sim 0 \%$ force with a flexed wrist. There was no effect of grip force when the wrist was extended $(P>0.05$; Fig. 6B). For key grip, there was a main effect of force $(P=0.008)$. The median nerve became more circular as force increased $(P<0.05$; Fig. 6C; Table 2$)$.

[INSERT FIGURE 6 ABOUT HERE.] 


\section{DISCUSSION}

The purpose of this study was to examine the combined effects of wrist posture, grip type, and grip force on median nerve cross-sectional area (CSA) and circularity at the carpal tunnel using ultrasound imaging. Our ultrasound measurements were reliable (as indicated by the good-to-excellent intraclass and interclass correlations) and consistent with previous ultrasound studies examining the carpal tunnel (Alemán et al., 2008; Filius et al., 2013; Loh and Muraki, 2015). We observed significant interactions between wrist posture, grip type, and grip force on median nerve circularity and mediolateral diameter. Increasing the grip force caused the median nerve to become elongated and less circular when the wrist was extended, but shorter and more circular when the wrist was flexed. Extending the wrist caused the median nerve to become more flattened during a four finger pinch grip when compared to the chuck grip or key grip. Overall, changes to the grip force, wrist posture, and/or grip type affected the shape of the median nerve, but not the CSA of the nerve.

Previous ultrasound studies have reported conflicting results on whether median nerve CSA is altered in different wrist postures or with different grips. Loh and Muraki (2015) found that median nerve CSA decreased during passive wrist flexion and extension, while Wang (2014b) found no change in CSA during active wrist flexion or extension. CSA decreased at the extreme flexion angle during active index finger or thumb movement (van Doesburg et al., 2010). However, CSA did not change during full finger flexion or a forceful grip with all four fingers in a neutral wrist posture (Filius et al., 2013). In this study, we did not observe significant changes in median nerve CSA during active gripping in any wrist posture. While we may have found differences in CSA with a larger number of subjects, the mean difference between the flexed and neutral wrist postures in this study was only $\sim 0.3 \mathrm{~mm}^{2}$. By contrast, previous work 
reported differences of $\sim 1.4 \mathrm{~mm}^{2}$ between wrist postures (Loh and Muraki, 2014, 2015). Compression of the median nerve may transversely displace the internal contents of the nerve (Topp and Boyd, 2006). The endoneurial fluid is displaced longitudinally within the nerve over a few minutes, leading to decreased median nerve CSA at the site of compression and increased median nerve CSA at the edges of compression (Dyck et al., 1990). Differences in the experimental methods of prior studies (e.g. active vs. passive tasks) could have affected the location of nerve compression, and the location and timing of image acquisition. These differences may explain the conflicting results.

Measurements of the shape and deformation of the median nerve may be more useful than CSA to understand the contact forces on the median nerve during dynamic tasks. When the wrist was flexed, the length of the long axis decreased while the short axis length increased (i.e. more circular), but during wrist extension, the long axis increased in length and the short axis decreased (i.e. less circular). These results are in agreement with prior work measuring changes in the long and short axes of the median nerve (Loh and Muraki, 2015) and nerve circularity (Wang et al., 2014b) with differing wrist postures. The current results demonstrate that nerve deformation increased with grip force, and the greatest deformation occurred when gripping was coupled with non-neutral wrist postures. In particular, the combination of wrist flexion and high tendon tension increases the force between flexor tendons and the median nerve (Keir and Wells, 1999). This explains why the largest changes in the shape of the median nerve were observed in wrist flexion when producing a 50\% grip force.

At the carpal tunnel, the median nerve lies between the digital flexor tendons and the transverse carpal ligament where it may be subjected to hydrostatic (Keir et al., 1998) or contact (Ko and Brown, 2007) compression forces and friction during wrist and finger movements 
(Sucher, 2009). Flexing or extending the wrist decreases the volume (Mogk and Keir, 2009) in the carpal tunnel and increases the force between tendons and adjacent structures (Armstrong and Chaffin, 1979). Prolonged compression can restrict blood flow leading to nerve pathology (Topp and Boyd, 2006). However, the development of CTS is associated with intermittent or repetitive movements rather than prolonged static postures. The median nerve may move longitudinally and transversely in the carpal tunnel during these activities (Filius et al., 2015; Lopes et al., 2011). In dynamic tasks, compressive forces might increase transverse or frictional forces acting on the nerve during tendon/nerve movement. During wrist flexion, these forces may displace the nerve posteriorly (Wang et al., 2014a). Although we did not directly measure nerve displacement, we observed that the median nerve initially became flattened and elongated during wrist flexion before displacing posterioulnarly where it became more circular. However, when the wrist was extended, the median nerve remained abutted to the transverse carpal ligament where it became more flattened as flexor tendons pressed against it. Carpal tunnel syndrome is characterized by fibrosis of the median nerve which may limit natural nerve motion within the carpal tunnel (Nakamichi and Tachibana, 1995) and lead to greater nerve deformation. Further work is needed to quantify nerve displacement and deformation in transverse and longitudinal planes during prehensile activities. The current observations emphasize that the mechanical stresses placed on the median nerve are complex and varied. Furthermore, changing the sequence of joint/muscle activity may alter these stresses (Topp and Boyd, 2006). For example, grasping and then flexing the wrist is likely to affect the nerve differently than flexing the wrist and then grasping. These effects need to be taken into account in order to adequately understand the development of CTS. 
The three grips tested in this study differentially affected the shape of the median nerve. We expected the chuck grip to have a large effect on nerve circularity because it primarily involved the first three digits whose tendons lie adjacent to the nerve. However, the four finger pinch grip had the largest effect on nerve circularity and axis length. Although, the grip force during four-finger pinch was $\sim 10 \%$ higher compared to chuck grip, it is unlikely that the different effects on the median nerve shape were due to differences in force. For example, the key grip force was $\sim 25 \%$ greater than the four finger pinch and chuck grip, but the effects of key grip on the median nerve were small. Instead, it is likely that differences between grip types were caused by different contact forces between the tendons and the median nerve in each grip. The four finger pinch grip involved forces applied at each fingertip, and thus all four tendons of the deep digital flexor muscle were under tension. By contrast, the key grip required relatively little tension in the finger flexor tendons. Thus, the mechanical interaction between the median nerve and the immediately adjacent tendons was dependent on the forces applied by all digits.

This study had several limitations. The current analysis was limited to measuring the cross section of the median nerve at the proximal carpal tunnel because the configuration of the hand during gripping limited the ability to obtain images of the nerve more distally in the carpal tunnel. Additionally, MRI studies demonstrate that the cut angle of cross-sectional images affects shape and area measurements (Mogk and Keir, 2008; Mogk and Keir, 2007). Ultrasonic images are more conducive to active tasks than MRI, but the precise angle of the ultrasound transducer relative to the median nerve cannot be ensured or corrected post hoc. In spite of this limitation, median nerve cross sectional ultrasound measurements have been gaining ground as clinically informative measures of disease (Filius et al., 2015). The ultrasound transducer was consistently placed in the current study at an angle of approximately 90 degrees to the forearm to obtain an 
axial slice and minimize errors associated with the cut angle. However, as with other ultrasound studies, it was not possible to ensure perfect axial orientation of the images relative to the nerve. Finally, anatomical variations can make it difficult to identify the median nerve in some individuals. For example, one subject with a bifurcated nerve was excluded from this study because it was not possible to analyze both sides of the nerve in all conditions. In spite of these challenges, ultrasound imaging has shown promising results in describing the shape and movement characteristics of healthy median nerves (Loh and Muraki, 2014, 2015; van Doesburg et al., 2010) and identifying pathologic nerves (Cartwright et al., 2013; Filius et al., 2015). This study only examined the wrists of healthy subjects. There may be different findings in people with people with CTS. Further, it is possible that in people who will develop CTS the median nerve moves differently than in typical healthy subjects. Future research will be aimed at identifying differences between healthy individuals and those with CTS.

The results of this study support evidence from epidemiology (Palmer et al., 2007) modeling (Armstrong and Chaffin, 1979), and other ultrasound studies (Loh and Muraki, 2015) that wrist flexion and extension can increase the likelihood of nerve injury. The combination of grasping with wrist flexion and extension increases deformation of the median nerve and may exacerbate risk in these wrist postures. These findings support the practice of immobilizing the wrist as a treatment or preventive strategy. The type of grip used may also be important when assessing the risk of median nerve injury as different grip types cause different interactions between the median nerve and surrounding tendons. Further work is needed to understand the forces on the median nerve during deformation and how this relates to the progression of pathology. These observations from healthy subjects provide normative data which can be compared to data collected from people with carpal tunnel syndrome in future studies. 


\section{ACKNOWLEDGEMENTS}

Funding was provided by a $\mathrm{xxx}$ graduate student research grant from $\mathrm{xxx}$. $\mathrm{Xxx}$ is supported by the xxx of the National Institutes of Health under Award Number xxx. We would like to thank $\mathrm{xxx}$, and $\mathrm{xxx}$ for their help with data collection and analysis.

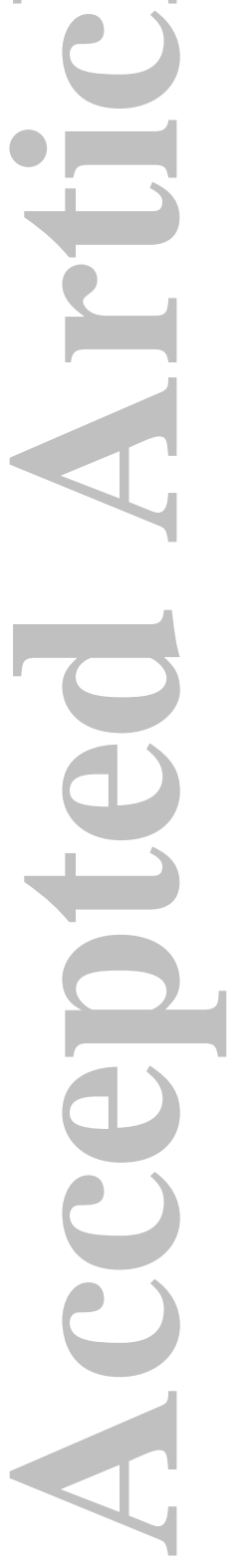

John Wiley and Sons, Inc. 


\section{REFERENCES}

Alemán L, Berná JD, Reus M, Martínez F, Doménech-Ratto G, Campos M. 2008.

Reproducibility of sonographic measurements of the median nerve. J Ultrasound Med 27:193-197.

An KN, Chao EY, Cooney WP, Linscheid RL. 1985. Forces in the normal and abnormal hand. J Orthop Res 3:202-211.

Armstrong T, Dale AM, Franzblau A, Evanoff BA. 2008. Risk factors for carpal tunnel syndrome and median neuropathy in a working population. J Occup Environ Med 50:1355-1364.

Armstrong TJ, Chaffin DB. 1979. Some biomechanical aspects of the carpal tunnel. J Biomech 12:567-570.

Bonfiglioli R, Mattioli S, Fiorentini C, Graziosi F, Curti S, Violante FS. 2007. Relationship between repetitive work and the prevalence of carpal tunnel syndrome in part-time and full-time female supermarket cashiers: a quasi-experimental study. Int Arch Occup Environ Health 80:248-253.

Cartwright MS, Walker FO, Blocker JN, Schulz MR, Arcury TA, Grzywacz JG, Mora D, Chen H, Marín AJ, Quandt SA. 2013. Ultrasound for carpal tunnel syndrome screening in manual laborers. Muscle Nerve 48:127-131.

Clark BD, Al-Shatti TA, Barr AE, Amin M, Barbe MF. 2004. Performance of a high-repetition, high-force task induces carpal tunnel syndrome in rats. J Orthop Sports Phys Ther 34:244-253.

Cox EP. 1927. A Method of Assigning Numerical and Percentage Values to the Degree of Roundness of Sand Grains. J Paleontol 1:179-183.

Dale AM, Harris-Adamson C, Rempel D, Gerr F, Hegmann K, Silverstein B, Burt S, Garg A,

Kapellusch J, Merlino L. 2013. Prevalence and incidence of carpal tunnel syndrome in US working populations: pooled analysis of six prospective studies. Scand J Work Environ Health 39:495.

Duncan I, Sullivan P, Lomas F. 1999. Sonography in the diagnosis of carpal tunnel syndrome. AJR Am J Roentgenol 173:681-684.

Dyck PJ, Lais AC, Giannini C, Engelstad JK. 1990. Structural alterations of nerve during cuff compression. Proc Natl Acad Sci U S A 87:9828-9832.

Filius A, Korstanje JW, Selles RW, Hovius SE, Slijper HP. 2013. Dynamic sonographic measurements at the carpal tunnel inlet: reliability and reference values in healthy wrists. Muscle Nerve 48:525-531.

Filius A, Scheltens M, Bosch HG, Van Doorn PA, Stam HJ, Hovius SE, Amadio PC, Selles RW. 2015. Multidimensional ultrasound imaging of the wrist: Changes of shape and displacement of the median nerve and tendons in carpal tunnel syndrome. J Orthop Res 33:1332-1340.

Fleiss JL. 1999. Reliability of Measurement. In: The Design and Analysis of Clinical Experiments: John Wiley \& Sons, Inc. p 1-32.

Goss BC, Agee JM. 2010. Dynamics of intracarpal tunnel pressure in patients with carpal tunnel syndrome. J Hand Surg 35:197-206.

Keir PJ, Bach JM, Hudes M, Rempel DM. 2007. Guidelines for wrist posture based on carpal tunnel pressure thresholds. Hum Factors 49:88-99. 
Keir PJ, Bach JM, Rempel DM. 1998. Fingertip loading and carpal tunnel pressure: differences between a pinching and a pressing task. J Orthop Res 16:112-115.

Keir PJ, Wells RP. 1999. Changes in geometry of the finger flexor tendons in the carpal tunnel with wrist posture and tendon load: an MRI study on normal wrists. Clin Biomech 14:635-645.

Ko C, Brown TD. 2007. A fluid-immersed multi-body contact finite element formulation for median nerve stress in the carpal tunnel. Comput Methods Biomech Biomed Engin 10:343-349.

Kociolek AM, Tat J, Keir PJ. 2015. Biomechanical risk factors and flexor tendon frictional work in the cadaveric carpal tunnel. J Biomech 48:449-455.

Levine DW, Simmons BP, Koris MJ, Daltroy LH, Hohl GG, Fossel AH, Katz JN. 1993. A self-

administered questionnaire for the assessment of severity of symptoms and functional

status in carpal tunnel syndrome. J Bone Joint Surg Am 75:1585-1592.

Li Z-M, Latash M, Zatsiorsky V. 1998. Force sharing among fingers as a model of the redundancy problem. Exp Brain Res 119:276-286.

Loh PY, Muraki S. 2014. Effect of wrist deviation on median nerve cross-sectional area at proximal carpal tunnel level. Iran J Public Health 43:180-185.

Loh PY, Muraki S. 2015. Effect of wrist angle on median nerve appearance at the proximal carpal tunnel. PLoS One 10:e0117930.

Lopes MM, Lawson W, Scott T, Keir PJ. 2011. Tendon and nerve excursion in the carpal tunnel in healthy and CTD wrists. Clin Biomech 26:930-936.

McGorry RW, Fallentin N, Andersen JH, Keir PJ, Hansen TB, Pransky G, Lin J-H. 2014. Effect of grip type, wrist motion, and resistance level on pressures within the carpal tunnel of normal wrists. J Orthop Res 32:524-530.

Mogk JP, Keir PJ. 2008. Wrist and carpal tunnel size and shape measurements: effects of posture. Clin Biomech 23:1112-1120.

Mogk JPM, Keir PJ. 2007. Evaluation of the carpal tunnel based on 3-D reconstruction from MRI. J Biomech 40:2222-2229.

Mogk JPM, Keir PJ. 2009. The effect of landmarks and bone motion on posture-related changes in carpal tunnel volume. Clin Biomech 24:708-715.

Nakamichi K, Tachibana S. 1992. Transverse sliding of the median nerve beneath the flexor retinaculum. J Hand Surg 17:213-216.

Nakamichi K, Tachibana S. 1995. Restricted Motion of the Median Nerve in Carpal Tunnel Syndrome. J Hand Surg 20:460-464.

Palmer KT, Harris EC, Coggon D. 2007. Carpal tunnel syndrome and its relation to occupation: a systematic literature review. Occup Med (Lond) 57:57-66.

Presazzi A, Bortolotto C, Zacchino M, Madonia L, Draghi F. 2011. Carpal tunnel: Normal anatomy, anatomical variants and ultrasound technique. J Ultrasound 14:40-46.

Schneider CA, Rasband WS, Eliceiri KW. 2012. NIH Image to ImageJ: 25 years of image analysis. Nat Meth 9:671-675.

Signs-CTS. 2000. Tinel's Sign and Phalen's Maneuver: Physical Signs of Carpal Tunnel Syndrome. Hosp Physician:39.

Silverstein BA, Fine LJ, Armstrong TJ. 1987. Occupational factors and carpal tunnel syndrome. Am J Ind Med 11:343-358.

Stapleton MJ. 2006. Occupation and Carpal Tunnel Syndrome. ANZ J Surg 76:494-496. 
Sucher BM. 2009. Ultrasound imaging of the carpal tunnel during median nerve compression. Curr Rev Musculoskelet Med 2:134-146.

Tinel J. 1945. Manifestations of "Pins and Needles Sensation" in Lesions of the Peripheral Nerves Presse Med 23:388-389.

Topp KS, Boyd BS. 2006. Structure and biomechanics of peripheral nerves: nerve responses to physical stresses and implications for physical therapist practice. Phys Ther 86:92-109. van Doesburg MHM, Henderson J, Mink van der Molen AB, An K-N, Amadio PC. 2012. Transverse Plane Tendon and Median Nerve Motion in the Carpal Tunnel: Ultrasound Comparison of Carpal Tunnel Syndrome Patients and Healthy Volunteers. PLoS One 7:e37081.

van Doesburg MHM, Yoshii Y, Villarraga HR, Henderson J, Cha SS, An K-N, Amadio PC.

2010. Median nerve deformation and displacement in the carpal tunnel during index finger and thumb motion. J Orthop Res 28:1387-1390.

Wang Y, Filius A, Zhao C, Passe SM, Thoreson AR, An K-N, Amadio PC. 2014a. Altered Median Nerve Deformation and Transverse Displacement during Wrist Movement in Patients with Carpal Tunnel Syndrome. Acad Radiol 21:472-480.

Wang Y, Zhao C, Passe SM, Filius A, Thoreson AR, An K-N, Amadio PC. 2014b. Transverse ultrasound assessment of median nerve deformation and displacement in the human carpal tunnel during wrist movements. Ultrasound Med Biol 40:53-61.

Yoshii Y, Villarraga HR, Henderson J, Zhao C, An KN, Amadio PC. 2009. Ultrasound assessment of the displacement and deformation of the median nerve in the human carpal tunnel with active finger motion. J Bone Joint Surg Am 91:2922-2930.

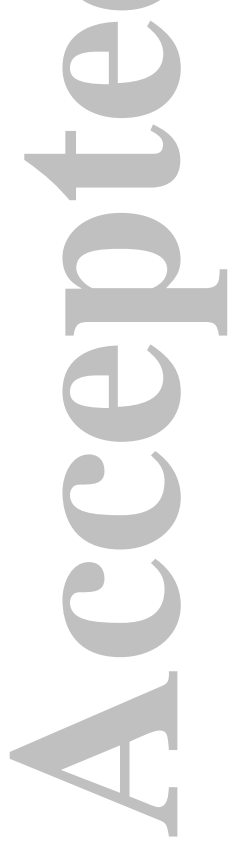




\section{Figure Captions}

Figure 1. Three grip types were used. Subjects grasped the dynamometer with the distal pad of the thumb and opposed thumb force using $\mathbf{A}$ ) four finger pinch grip: the distal pads of all four fingers, B) chuck grip: the distal pads of the index and middle fingers only, C) key (lateral pinch) grip: the lateral aspect of the proximal interphalangeal joint of the index finger.

Figure 2. Ultrasound wrist images were analyzed using a tracing method. The median nerve was identified by the white outer boundary. The polygon selection tool was used to outline the median nerve, and the cross-sectional area and circularity measurements were obtained using ImageJ software.

Figure 3. Average maximum grip force in each wrist posture and grip type. Error bars are the $95 \%$ confidence interval. * indicates a significant difference from the neutral wrist posture.

Figure 4. The cross-sectional area during A) four finger pinch, B) chuck, and C) key grips. The dashed line shows the average area for wrist neutral with no grip. Error bars are the 95\% confidence interval.

Figure 5. Mediolateral (TOP), and anteroposterior (BOTTOM) diameters of the median nerve in each wrist posture during A) four finger pinch, B) chuck, and C) key grips. Error bars are the $95 \%$ confidence interval. * indicates a significant difference from the neutral wrist posture. $\dagger$ indicates a significant difference from $0 \%$ force.

Figure 6. TOP) Median nerve circularity during A) four finger pinch, B) chuck, and C) key grips. The dashed line shows the average circularity for wrist neutral with no grip. Error bars are the $95 \%$ confidence interval. BOTTOM) Ultrasound images during four finger pinch grip show a flattened median nerve in the extended wrist posture (D) and a circular nerve in the flexed wrist posture $(\mathbf{E}) . *$ indicates a significant difference from the neutral wrist posture. $\dagger$ indicates a significant difference from $0 \%$ force. 
Table 1. Intraclass correlation coefficients, $\operatorname{ICC}(2,1)$.

\begin{tabular}{l|cccc} 
Measure & CSA & Circularity & ML Axis & AP Axis \\
\hline Intra-rater & 0.940 & 0.921 & 0.873 & 0.957 \\
Inter-rater & 0.896 & 0.729 & 0.773 & 0.893
\end{tabular}

John Wiley and Sons, Inc.

This article is protected by copyright. All rights reserved. 
Table 2. Average (standard deviation) of cross-sectional area (CSA), mediolateral diameter (ML Axis), anteroposterior diameter (AP axis), and circularity for each condition.

\begin{tabular}{|c|c|c|c|c|c|c|}
\hline Grip Type & Wrist Posture & Force & CSA & ML Axis & AP Axis & Circularity \\
\hline \multirow{3}{*}{ None } & Neutral & NA & $7.54(1.47)$ & $5.69(0.93)$ & $2.06(0.33)$ & $0.57(0.11)$ \\
\hline & Flexed & NA & $7.20(1.20)$ & $5.17(0.63)$ & $2.40(0.55)$ & $0.60(0.09)$ \\
\hline & Extended & NA & $7.23(1.23)$ & $5.80(0.94)$ & $1.89(0.23)$ & $0.55(0.10)$ \\
\hline \multirow{9}{*}{ Pinch } & \multirow{3}{*}{ Neutral } & 0 & $7.33(1.33)$ & $5.87(0.89)$ & $1.92(0.38)$ & $0.54(0.09)$ \\
\hline & & 25 & $7.42(1.16)$ & $5.94(0.98)$ & $2.00(0.29)$ & $0.53(0.08)$ \\
\hline & & 50 & $7.27(1.24)$ & $5.87(1.00)$ & $1.97(0.34)$ & $0.53(0.08)$ \\
\hline & \multirow{3}{*}{ Flexed } & 0 & $7.26(1.18)$ & $5.27(0.81)$ & $2.46(0.54)$ & $0.58(0.08)$ \\
\hline & & 25 & $7.22(1.19)$ & $4.57(0.76)$ & $2.74(0.49)$ & $0.65(0.08)$ \\
\hline & & 50 & $7.32(1.12)$ & $4.43(1.00)$ & $2.84(0.54)$ & $0.68(0.10)$ \\
\hline & \multirow{3}{*}{ Extended } & 0 & $7.23(1.46)$ & $5.88(1.04)$ & $1.85(0.25)$ & $0.54(0.10)$ \\
\hline & & 25 & $7.20(1.35)$ & $6.05(0.90)$ & $1.77(0.19)$ & $0.51(0.07)$ \\
\hline & & 50 & $7.08(1.44)$ & $6.11(1.08)$ & $1.80(0.27)$ & $0.50(0.10)$ \\
\hline \multirow{9}{*}{ Chuck } & \multirow{3}{*}{ Neutral } & 0 & $7.25(1.08)$ & $5.78(0.83)$ & $1.89(0.25)$ & $0.54(0.10)$ \\
\hline & & 25 & $7.10(1.09)$ & $5.77(0.91)$ & $1.91(0.26)$ & $0.54(0.09)$ \\
\hline & & 50 & $7.05(0.93)$ & $5.74(0.97)$ & $1.93(0.31)$ & $0.54(0.13)$ \\
\hline & \multirow{3}{*}{ Flexed } & 0 & $7.31(1.30)$ & $5.31(0.73)$ & $2.53(0.48)$ & $0.58(0.07)$ \\
\hline & & 25 & $7.33(1.37)$ & $4.90(0.96)$ & $2.73(0.54)$ & $0.63(0.11)$ \\
\hline & & 50 & $7.19(1.32)$ & $4.69(0.93)$ & $2.75(0.52)$ & $0.65(0.11)$ \\
\hline & \multirow{3}{*}{ Extended } & 0 & $7.18(1.37)$ & $5.44(0.89)$ & $2.08(0.31)$ & $0.59(0.10)$ \\
\hline & & 25 & $7.19(1.24)$ & $5.44(0.89)$ & $2.01(0.32)$ & $0.59(0.10)$ \\
\hline & & 50 & $7.12(1.32)$ & $5.57(0.93)$ & $1.98(0.32)$ & $0.56(0.10)$ \\
\hline \multirow{9}{*}{ Key } & \multirow{3}{*}{ Neutral } & 0 & $7.29(1.14)$ & $5.68(0.88)$ & $1.98(0.22)$ & $0.56(0.09)$ \\
\hline & & 25 & $7.26(1.23)$ & $5.55(0.72)$ & $2.04(0.26)$ & $0.57(0.08)$ \\
\hline & & 50 & $7.30(1.20)$ & $5.51(0.78)$ & $2.07(0.29)$ & $0.58(0.09)$ \\
\hline & \multirow{3}{*}{ Flexed } & 0 & $7.46(1.24)$ & $5.49(1.10)$ & $2.40(0.59)$ & $0.56(0.11)$ \\
\hline & & 25 & $7.38(1.38)$ & $5.21(1.13)$ & $2.46(0.54)$ & $0.59(0.12)$ \\
\hline & & 50 & $7.51(1.42)$ & $5.19(1.11)$ & $2.56(0.62)$ & $0.59(0.12)$ \\
\hline & \multirow{3}{*}{ Extended } & 0 & $7.22(1.15)$ & $5.73(0.98)$ & $2.06(0.25)$ & $0.55(0.10)$ \\
\hline & & 25 & $7.19(1.17)$ & $5.48(0.93)$ & $2.15(0.30)$ & $0.57(0.09)$ \\
\hline & & 50 & $7.21(1.18)$ & $5.45(0.82)$ & $2.15(0.33)$ & $0.57(0.09)$ \\
\hline
\end{tabular}

John Wiley and Sons, Inc.

This article is protected by copyright. All rights reserved. 

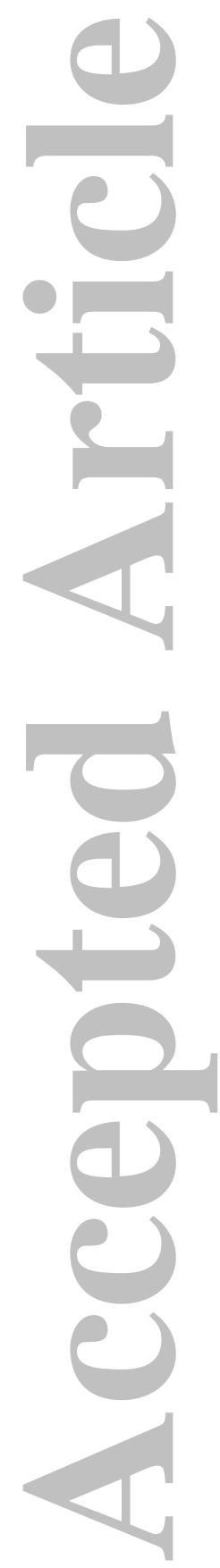

John Wiley and Sons, Inc.

This article is protected by copyright. All rights reserved. 

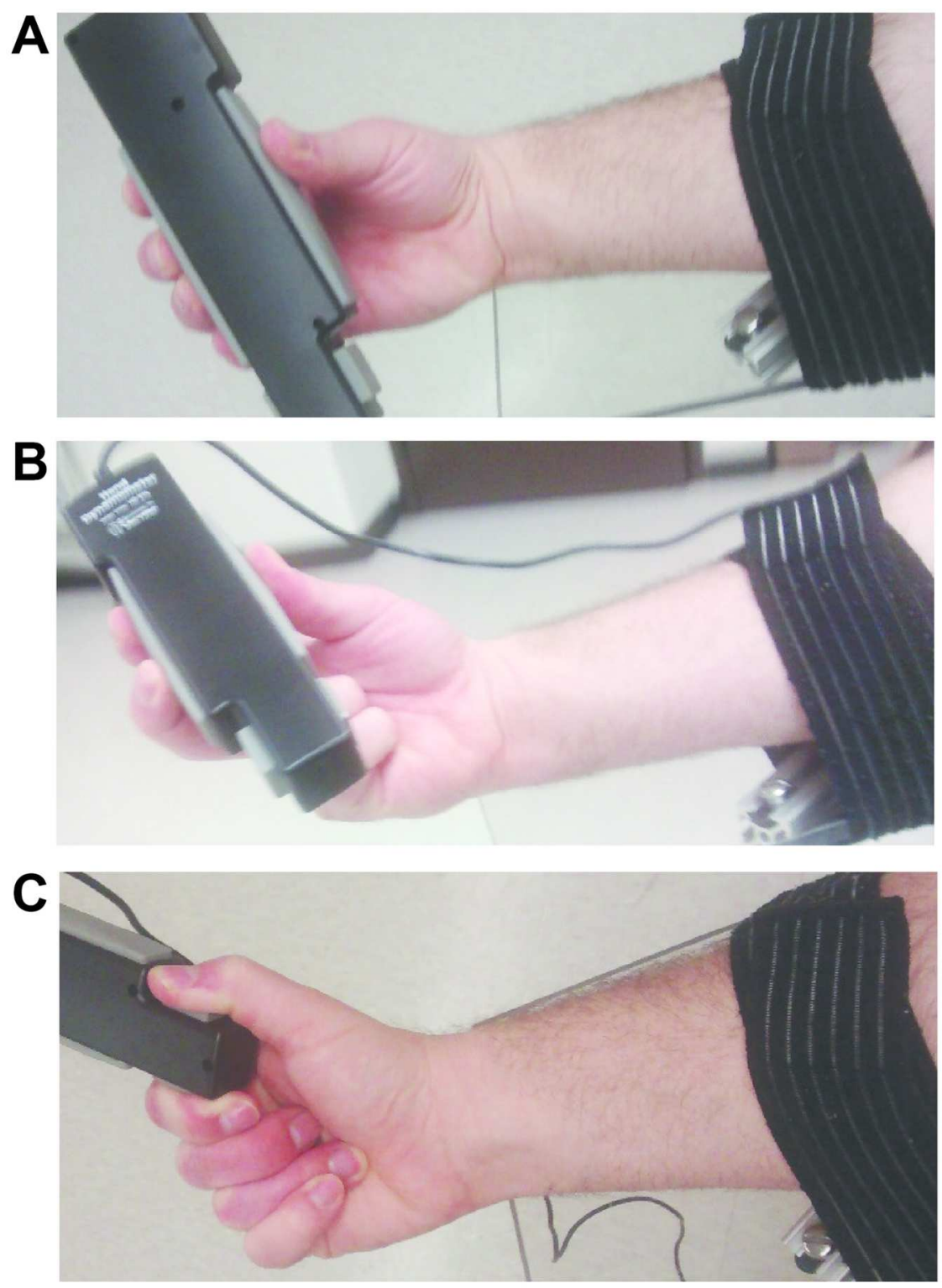

Figure 1. Three grip types were used. Subjects grasped the dynamometer with the distal pad of the thumb and opposed thumb force using A) four finger pinch grip: the distal pads of all four fingers, B) chuck grip: the distal pads of the index and middle fingers only, C) key (lateral pinch) grip: the lateral aspect of the proximal interphalangeal joint of the index finger.

Fig. 1

$114 \times 154 \mathrm{~mm}(300 \times 300 \mathrm{DPI})$

John Wiley and Sons, Inc.

This article is protected by copyright. All rights reserved. 

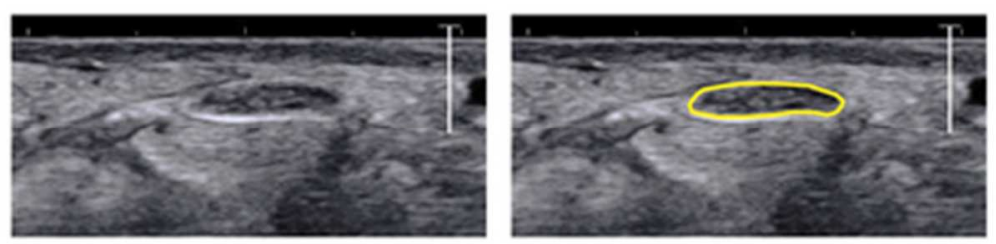

Figure 2. Ultrasound wrist images were analyzed using a tracing method. The median nerve was identified by the white outer boundary. The polygon selection tool was used to outline the median nerve, and the cross-sectional area and circularity measurements were obtained using Image] software.

Fig. 2

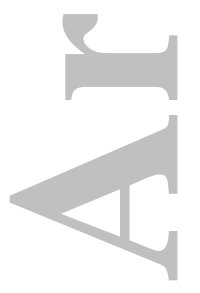

$32 \times 8 \mathrm{~mm}(300 \times 300 \mathrm{DPI})$

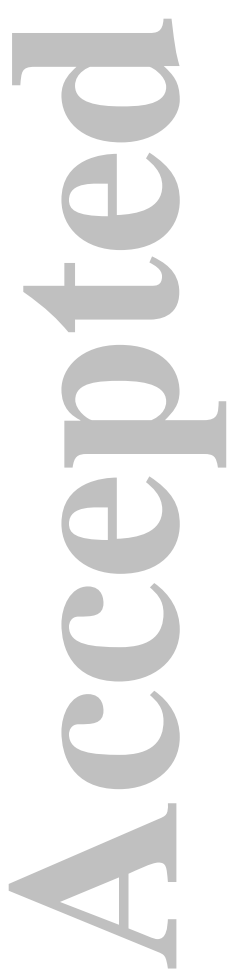

John Wiley and Sons, Inc.

This article is protected by copyright. All rights reserved. 


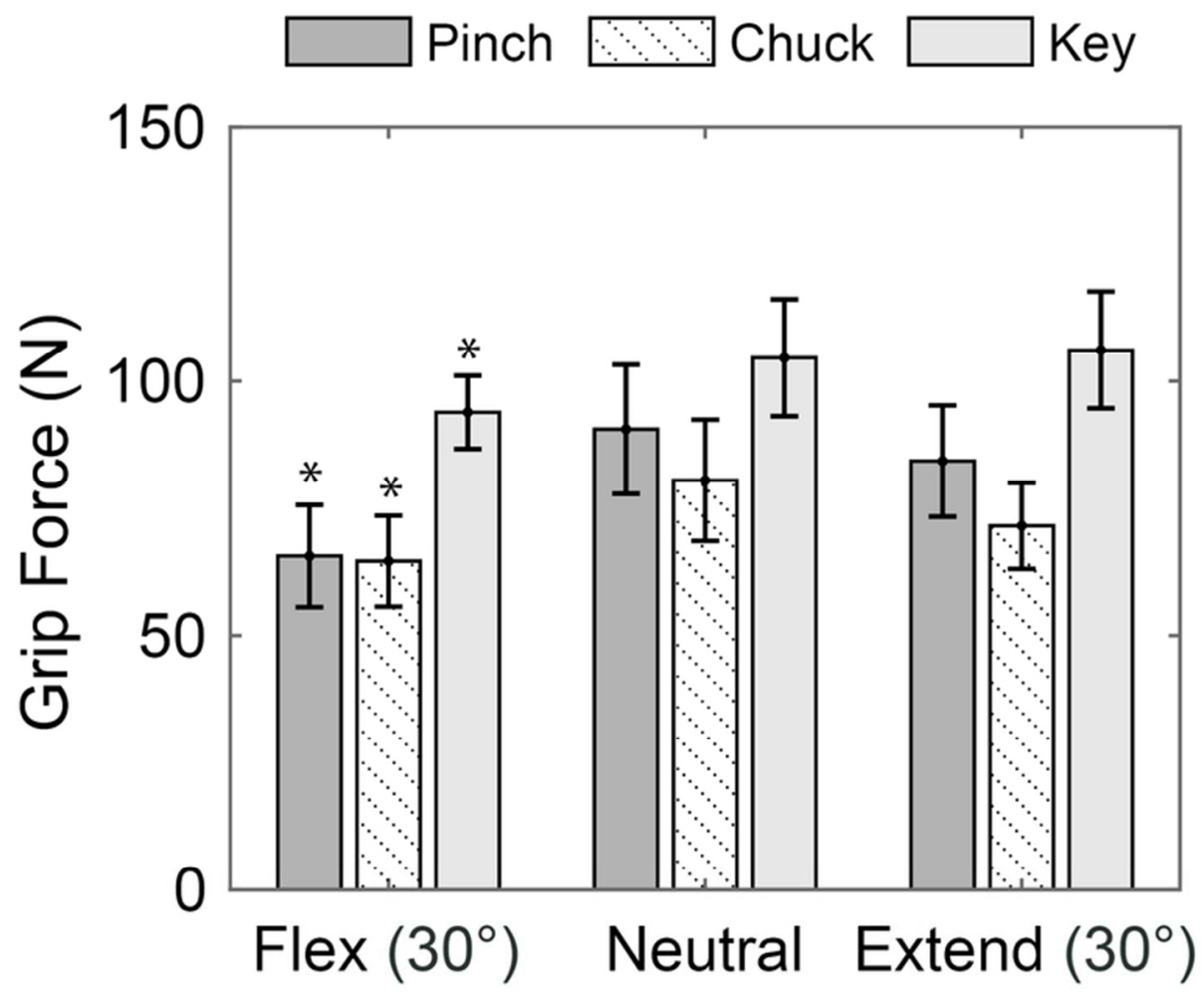

Figure 3. Average maximum grip force in each wrist posture and grip type. Error bars are the $95 \%$ confidence interval. * indicates a significant difference from the neutral wrist posture.

Fig. 3

$70 \times 55 \mathrm{~mm}(300 \times 300 \mathrm{DPI})$ 


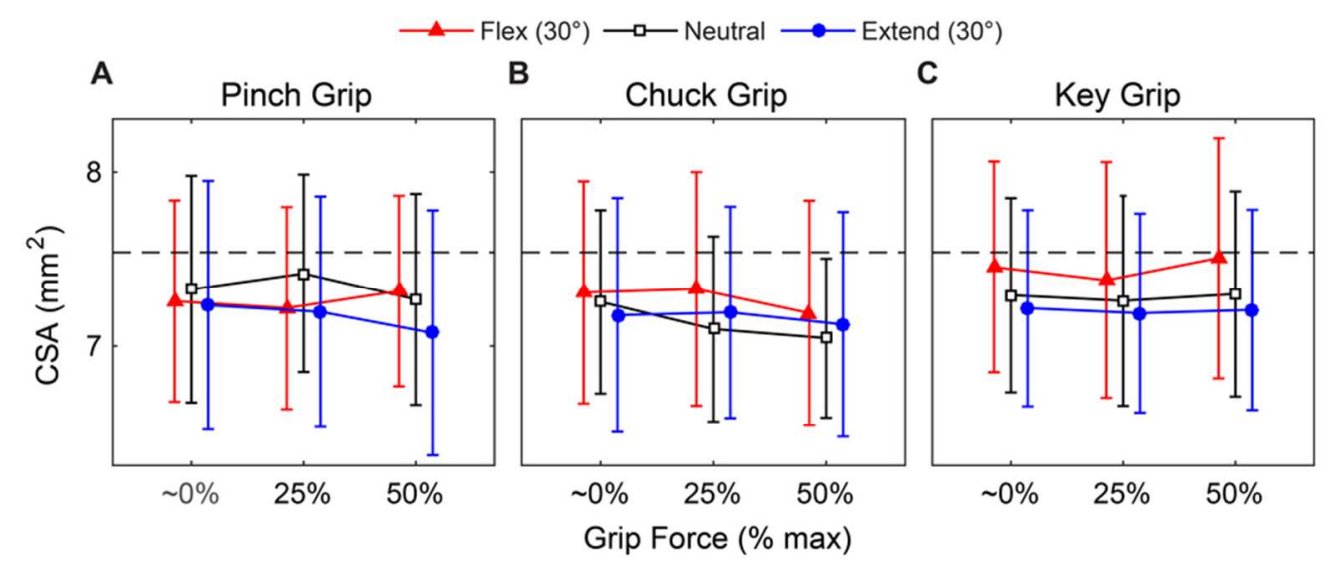

Figure 4. The cross-sectional area during A) four finger pinch, B) chuck, and C) key grips. The dashed line shows the average area for wrist neutral with no grip. Error bars are the $95 \%$ confidence interval.

Fig. 4

$79 \times 33 \mathrm{~mm}(300 \times 300 \mathrm{DPI})$

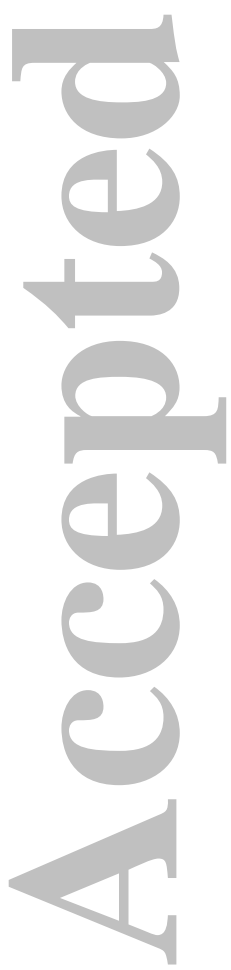

John Wiley and Sons, Inc.

This article is protected by copyright. All rights reserved. 

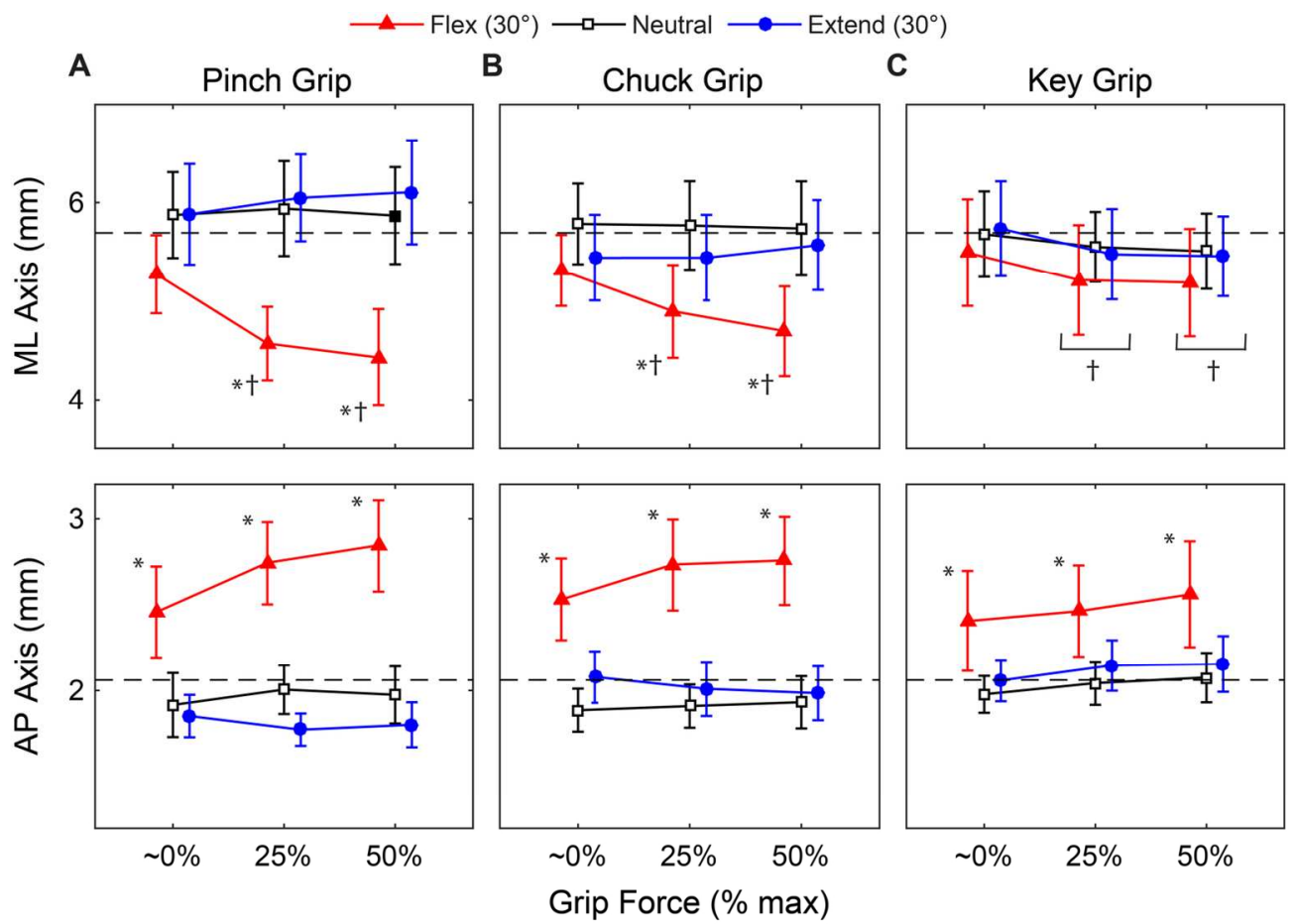

Figure 5. Mediolateral (TOP), and anteroposterior (BOTTOM) diameters of the median nerve in each wrist posture during A) four finger pinch, B) chuck, and C) key grips. Error bars are the $95 \%$ confidence interval. $*$ indicates a significant difference from the neutral wrist posture. + indicates a significant difference from 0 $\%$ force.

Fig. 5

$134 \times 95 \mathrm{~mm}(300 \times 300 \mathrm{DPI})$

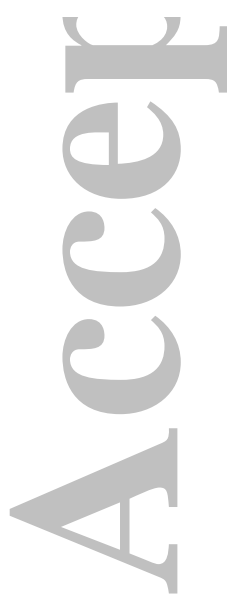

John Wiley and Sons, Inc.

This article is protected by copyright. All rights reserved. 


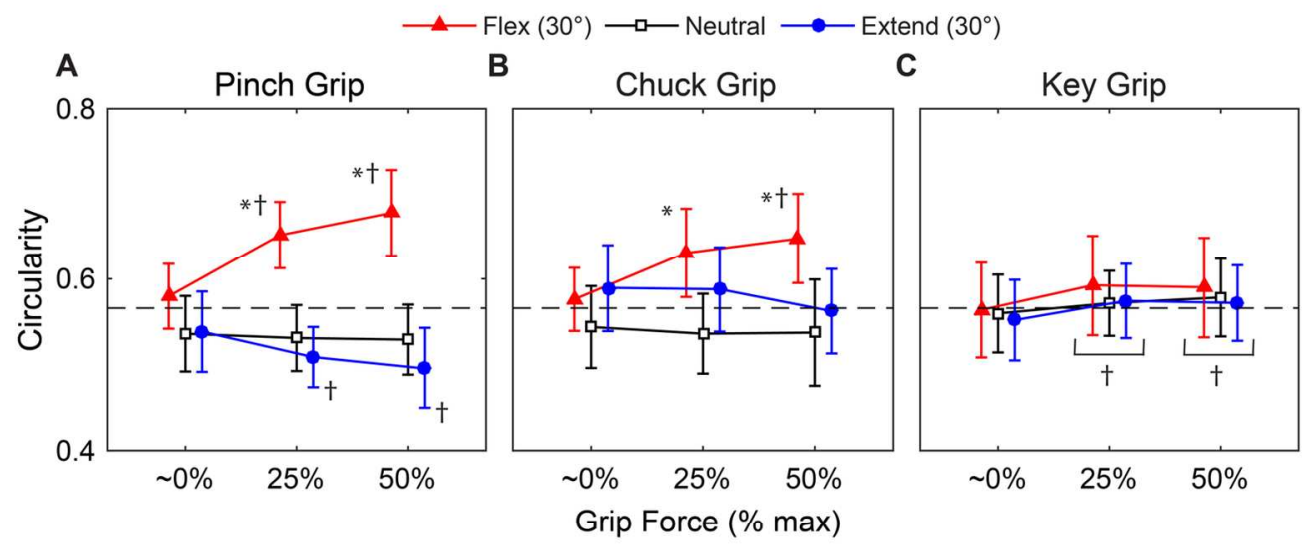

D

E
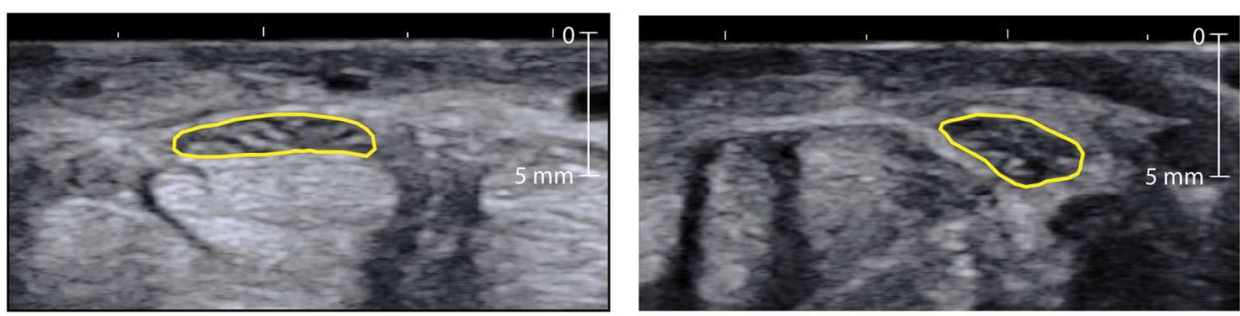

Figure 6. TOP) Median nerve circularity during A) four finger pinch, B) chuck, and C) key grips. The dashed line shows the average circularity for wrist neutral with no grip. Error bars are the $95 \%$ confidence interval. BOTTOM) Ultrasound images during four finger pinch grip show a flattened median nerve in the extended wrist posture (D) and a circular nerve in the flexed wrist posture (E). * indicates a significant difference from the neutral wrist posture. + indicates a significant difference from $0 \%$ force.

Fig. 6

$137 \times 98 \mathrm{~mm}(300 \times 300 \mathrm{DPI})$

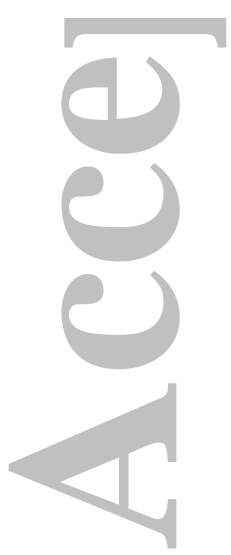

John Wiley and Sons, Inc.

This article is protected by copyright. All rights reserved. 